\title{
The Relative Contributions of Experiential Avoidance and Distress Tolerance to OC Symptoms
}

\author{
Shannon M. Blakey, Ryan J. Jacoby, Lillian Reuman and Jonathan S. Abramowitz \\ University of North Carolina at Chapel Hill, USA
}

\begin{abstract}
Background: Obsessive beliefs account for substantial (but not all) obsessive-compulsive (OC) symptoms. Intolerance of internal experiences (IIE), which encompasses the constructs of experiential avoidance (EA) and distress tolerance (DT), refers to difficulty managing unwanted thoughts, emotions, and other internal states, and might add to current explanatory models. Although IIE appears to be conceptually relevant to obsessive-compulsive (OC) symptoms, scant research has examined this relationship empirically. Aim: The present study examined the relative contributions of EA and DT as predictors of OC symptom dimensions. Method: A nonclinical sample $(n=496)$ completed self-report questionnaires measuring general distress, EA, DT and OC symptom dimensions. Results: All variables of interest were significantly (all $p \mathrm{~s} \leq .001$ ) correlated with one another, such that higher general distress, higher EA, and lower DT were associated with greater OC symptom severity for all symptom dimensions; however, only EA independently predicted obsessional symptoms, but not other OC symptom dimensions. Conclusions: One's willingness to endure (i.e. EA), rather than their ability to tolerate (i.e. DT) unpleasant internal experiences best predicts obsessional symptoms (i.e. obsessing) above and beyond general distress. Potential implications for understanding, assessing, and treating OC symptoms are discussed.
\end{abstract}

Keywords: Obsessive-compulsive disorder, obsessions, experiential avoidance, distress tolerance.

\section{Introduction}

Obsessive-compulsive disorder (OCD) is a heterogeneous psychological condition characterized by distressing, unwanted, intrusive thoughts, images, or impulses (obsessions) and behaviors performed to prevent feared consequences and/or reduce anxiety (e.g. compulsive rituals). Although various conceptual models have been proposed to account for the development and maintenance of OC symptoms (e.g. Salkovskis, 1999; Teasdale, 1974), research consistently provides support for the importance of maladaptive beliefs. The Obsessive-Compulsive Cognitions Working Group (OCCWG; 1997, 2001, 2003), for example, highlighted several types of maladaptive beliefs, such as inflated estimates of responsibility, over-importance of (and need to control) thoughts, overestimation of threat, intolerance of uncertainty, and perfectionism. Accumulated research indicates that such dysfunctional cognitions (i.e. "obsessive beliefs") are associated with OC symptoms in

Reprint requests to Jonathan Abramowitz, University of North Carolina at Chapel Hill, C.B. \# 3270 (Davie Hall), Chapel Hill, NC 27599, USA. E-mail: jabramowitz@unc.edu 
clinical and nonclinical samples, with correlations ranging between .14 and .41 after partialling out general distress (OCCWG, 2005; Wu and Carter, 2008). Nevertheless, obsessive beliefs do not fully account for OC symptoms. Consequently, investigators have begun to consider other psychological constructs that might also be associated with OC symptoms and potentially add explanatory power to existing models; as is the focus of the present study.

Broadly, the intolerance of internal experiences (IIE) refers to difficulty managing unwanted thoughts, emotions, and other internal states, a difficulty that is often observed among individuals with OCD (e.g. Forsyth, Eifert and Barrios, 2006; Robinson and Freeston, 2014; Twohig, 2009). IIE encompasses two phenomena: experiential avoidance (EA) and distress tolerance (DT). EA refers to the unwillingness to experience unpleasant emotions, thoughts or memories (Hayes, Wilson, Gifford, Follette and Strosahl, 1996). ${ }^{1}$ DT refers to one's capacity to endure aversive emotions that are experienced as unacceptable or unmanageable (Leyro, Zvolensky and Bernstein, 2010). Despite their conceptual overlap, key differences between these two constructs include the emphasis on being "willing and open" to unwanted internal experiences (i.e. EA; "I am willing to think about what would happen if my mom died in a car accident") versus simply being "able to endure" them (i.e. DT; "I can bear to think about my mom dying in a car accident"). Both EA and DT may play a role in the maintenance of OC symptoms in that they each drive efforts to control obsessional anxiety and unwanted thoughts (Robinson and Freeston, 2014), usually through the use of covert or overt strategies (i.e. compulsive rituals). Although such rituals might temporarily reduce the distress associated with obsessions, they paradoxically exacerbate OC symptoms in the long term (e.g. Wetterneck, Steinberg and Hart, 2014).

Despite the apparent conceptual relevance of IIE to OC symptoms, there is a shortage of empirical research on this topic; most attention has been focused on obsessive beliefs as predictors of OC symptoms, and investigators have only recently begun to even consider alternate empirical directions. To date, the findings from research on EA and DT with respect to OC symptoms have been mixed, perhaps due to conceptual and methodological differences across studies (e.g. use of different measures of EA), as well as differences in data analytic approaches (e.g. using global versus dimensional OC symptom measures). For instance, although Briggs and Price (2009) reported significant associations between EA and both global OC symptoms and obsessive beliefs (e.g. inflated responsibility) in their unselected sample, Abramowitz, Lackey and Wheaton (2009) found that EA did not contribute significantly to the prediction of any OC symptom dimensions over and above the contribution of general distress and obsessive beliefs in an analogue sample. Using an outdated measure of EA (Bond et al., 2011; Wolgast, 2014), Manos and colleagues (2010) replicated Abramowitz and colleagues' findings while controlling for depression in a sample of OCD patients.

Findings regarding the importance of DT to OC symptoms are also inconclusive. Although Hezel, Riemann and McNally (2012) found that OCD patients had lower DT than nonclinical individuals, the authors neither controlled for negative affect (which has been associated with both DT and OCD symptoms; e.g. Ricciardi and McNally, 1995), nor examined the

\footnotetext{
1 "Psychological flexibility", the converse of EA, is a term often used in the Acceptance and Commitment Therapy (ACT) literature
} 
differential relationship of DT to OC symptom domains (e.g. contamination, symmetry). OCD is a heterogeneous condition, and it is possible that IIE is involved in some, but not all, presentations of OC symptoms. To this end, in two studies with nonclinical individuals, Cougle and colleagues $(2011,2012)$ found that lower DT was associated with obsessional symptoms, but not with other sorts of compulsive behaviors (e.g. checking, washing).

Still, other investigations call into question the stability of the relationship between DT and OC symptoms. For example, Macatee, Capron, Schmidt and Cougle (2013) found that low DT was associated with greater severity of obsessions in both clinical and nonclinical samples, but only in the context of greater life stress (i.e. life stressors moderated the DT-OC symptom relationship). Finally, although Keough, Riccardi, Timpano, Mitchell and Schmidt (2010) found that DT uniquely predicted multiple forms of anxiety (i.e. worry, social anxiety, panic concerns, and OCD symptoms), this relationship was weakest among patients with OCD relative to these other disorders. These mixed findings highlight the need for additional studies elucidating the relation of DT (and IIE more broadly) to the various OC symptom domains to advance this budding area of research.

Accordingly, the goal of the present study was to shed light on these relationships by replicating and extending previous work. In particular, we sought to understand the relative contributions of EA and DT in the prediction of OC symptom dimensions as no study has yet examined the unique effects of these constructs. That is, no statistical model to date has simultaneously accounted for both EA and DT in the prediction of the various dimensions of OC symptoms. Considering their theoretical relatedness and potential relevance to the etiology and treatment of OCD (Leyro et al., 2010; Robinson and Freeston, 2014), elucidating these relationships may help researchers prioritize indices of IIE when conceptualizing, assessing, and treating OCD. On the basis of existing theory and research, we hypothesized that IIE (i.e. both EA and DT) would predict both obsessions and neutralizing OC symptoms even after accounting for general distress.

\section{Method}

\section{Participants}

We tested our hypotheses using an unselected sample given the continuous (i.e. dimensional) expression of EA (e.g. Chawla and Ostafin, 2007), DT (Bernstein, Zvolensky, Vujanovic and Moos, 2009), and OC symptomatology (e.g. Rachman and de Silva, 1978; Salkovskis and Harrison, 1984) across clinical and non-clinical individuals, along with the costs (both financial and temporal) of recruiting clinical samples large enough for meaningful tests of our hypotheses. Research also indicates that associations between most OC-related phenomena are constant across clinical and nonclinical samples given that the main differences between such samples are quantitative, rather than qualitative (e.g. Abramowitz et al., 2014).

Our final sample included 496 undergraduate psychology students at the University of North Carolina at Chapel Hill (UNC-CH) who participated in this study for course credit. The sample was mostly female $(n=321 ; 64.6 \%)$ with a mean age of 19.10 years old $(S D=$ 1.20 , range 18 to 25$)$. The majority of participants identified as white $(n=389 ; 74 \%)$, with $10 \%(n=49)$ identifying as black, $9 \%(n=45)$ identifying as Asian, $4 \%(n=21)$ identifying as Hispanic/Latino, and $2 \%(n=12)$ identifying with another racial/ethnic group. 


\section{Procedure}

Participation was open to all undergraduate students enrolled in introductory psychology courses. Upon electronically consenting to participate, respondents were directed to a survey link hosted by Qualtrics, a secure online survey development tool. Participants completed the measures described below in random order, followed by a demographics questionnaire. Two distractor items (e.g. "please answer Always True for this item") were also included among the measures to increase the probability that only valid responses from attentive participants would be included in analyses. This study was approved by the UNC-CH Institutional Review Board.

\section{Measures}

Obsessive-Compulsive Inventory-Revised (OCI-R; Foa et al., 2002). The OCI-R is an 18item self-report measure of OC symptom severity. Participants rate the extent to which they were distressed or bothered by 18 OC symptoms during the past month on a 0 (not at all) to 4 (extremely) scale; higher scores indicate greater symptom severity. Items load onto six subscales (five of which were used for the current study): washing, checking, obsessing, neutralizing, and ordering. Because hoarding is no longer considered an OC symptom (hoarding was reclassified as a separate disorder in DSM-5; APA, 2013), the hoarding subscale was excluded from analyses. The complete version of the OCI-R has demonstrated good internal consistency, test-retest reliability, and convergent and divergent validity in previous work. Internal consistencies for the assessed OCI-R scales ranged from good to excellent $\left(\alpha_{\text {check }}=.71 ; \alpha_{\text {order }}=.86 ; \alpha_{\text {neut }}=.70 ; \alpha_{\text {wash }}=.78 ; \alpha_{\text {obsess }}=.82\right)$ in the current sample.

Depression Anxiety Stress Scales-21 (DASS-21; Antony, Bieling, Cox, Enns and Swinson, 1998). The DASS-21 is a short-form version of the 42-item DASS (Lovibond and Lovibond, 1995) that assesses subjective distress over the past week along three subscales: depression, anxiety, and stress. Participants rate how each of the 21 statements (e.g. "I found it hard to wind down") applies to them on a 0 (rarely) to 4 (very much, or most of the time) scale; higher scores indicate greater general distress. The DASS-21 has demonstrated good reliability and construct validity in both clinical and non-clinical samples (Henry and Crawford, 2005; Page, Hooke and Morrison, 2007). The DASS-21 showed excellent internal consistency $(\alpha=.90)$ in the current sample.

Acceptance and Action Questionnaire-II (AAQ-II; Bond et al., 2011). The AAQ-II is a 7-item revision of the original 10-item AAQ (Bond et al., 2011). The scale assesses psychological flexibility, which is the inverse of EA and is the core construct of the ACT model of psychopathology (Hayes et al., 2006). Participants rate their agreement with each of the 7 statements (e.g. "I'm afraid of my feelings") on a 1 (never true) to 7 (always true) scale; higher scores indicate lesser psychological flexibility (i.e. more EA and pathology). The AAQ-II has been shown to have a single factor and has demonstrated good psychometric properties as well as convergent, discriminant, and incremental validity in previous work. The AAQ-II showed excellent internal consistency $(\alpha=.92)$ in the current sample.

Distress Tolerance Scale (DTS; Simons and Gaher, 2005). The DTS is a 15-item measure of one's ability to withstand aversive psychological states. Participants rate the extent to which they agree with each statement (e.g. "Feeling distressed or upset is unbearable to me") on a 1 
Table 1. Descriptive statistics

\begin{tabular}{lrrrrrr}
\hline & $M$ & $(S D)$ & Min & Max & Skew & Kurtosis \\
\hline OCI-R & & & & & & \\
$\quad$ Checking & 1.85 & $(2.15)$ & 0 & 12 & 1.93 & 4.53 \\
$\quad$ Ordering & 3.12 & $(2.86)$ & 0 & 12 & 1.00 & 0.50 \\
$\quad$ Neutralizing & 1.26 & $(2.01)$ & 0 & 12 & 2.15 & 5.22 \\
Washing & 1.44 & $(2.23)$ & 0 & 12 & 2.08 & 4.58 \\
$\quad$ Obsessing & 2.00 & $(2.37)$ & 0 & 11 & 1.40 & 1.58 \\
DASS-21 & 11.94 & $(8.82)$ & 0 & 48 & 1.17 & 1.36 \\
AAQ-II & 17.42 & $(8.42)$ & 7 & 49 & 0.81 & 0.17 \\
DTS & 52.42 & $(11.99)$ & 18 & 75 & -0.49 & -0.19 \\
\hline
\end{tabular}

Notes: OCI-R = Obsessive-Compulsive Inventory-Revised; DASS-21 = Depression Anxiety Stress Scales-21; AAQ-II = Acceptance and Action Questionnaire-II; DTS $=$ Distress Tolerance Scale

(strongly agree) to 5 (strongly disagree) scale; higher scores indicate greater distress tolerance (less pathology). The DTS has demonstrated good internal consistency, test-retest reliability, and discriminant validity in previous work. The DTS showed excellent internal consistency $(\alpha=.92)$ in the current sample.

\section{Results}

\section{Data screening}

Of the 497 participants who completed the survey, one did not pass both distractor items and was consequently excluded from further data analysis, bringing the final sample size to 496 . Data were then screened to assess concordance with statistical assumptions. No univariate outliers were detected and the distributions of scores on all of the study measures were free of significant skew (all values $<3$ ). Some positive kurtosis was observed in the data (kurtosis values for the OCI-R checking, neutralizing, and washing subscales ranged 4.5 to 5.2; see Table 1); however, these values were only slightly higher than the ideal kurtosis absolute value limit of 4, and Kline (2005) has argued that detrimental effects of positive kurtosis values up to 10 are negligible at $N \geq 100$. Consequently, to retain interpretive value, we elected not to apply a transformation to the data before conducting the analyses reported below.

Descriptive statistics for all study variables are shown in Table 1. In general, mean scores fell within the expected range for nonclinical samples. As can also be seen, ordering OC symptoms appeared to be the most common, followed by obsessional symptoms.

\section{Zero-order correlations}

Two-tailed zero-order correlations were conducted to examine the relationship between OC symptom severity, general distress, EA, and DT. To control for multiple comparisons we used a Bonferroni corrected critical $\alpha=.002(.05 / 28)$. As seen in Table 2, all variables were significantly (all $p \mathrm{~s} \leq .001$ ) correlated with one another (ranging in magnitude from -.50 to 
Table 2. Zero-order correlations between study measures

\begin{tabular}{|c|c|c|c|c|c|c|c|c|}
\hline & & 1 & 2 & 3 & 4 & 5 & 6 & 7 \\
\hline & \multicolumn{8}{|l|}{ OCI-R } \\
\hline 1. & Checking & - & & & & & & \\
\hline 2. & Ordering & .52 & - & & & & & \\
\hline 3. & Neutralizing & .59 & .50 & - & & & & \\
\hline 4. & Washing & .48 & .49 & .46 & - & & & \\
\hline 5. & Obsessing & .47 & .33 & .40 & .35 & - & & \\
\hline 6. & DASS-21 & .37 & .22 & .27 & .16 & .48 & - & \\
\hline 7. & AAQ-II & .36 & .22 & .22 & .19 & .55 & .60 & - \\
\hline 8. & DTS & -.28 & -.19 & -.21 & -.15 & -.39 & -.50 & -.56 \\
\hline
\end{tabular}

Notes: OCI-R = Obsessive-Compulsive Inventory-Revised; DASS-21 = Depression Anxiety Stress Scales-21; AAQ-II = Acceptance and Action Questionnaire-II; DTS = Distress Tolerance Scale. All values significant at $p \leq .001$

$.60)$, such that higher general distress, higher EA, and lower DT were associated with greater OC symptom severity.

\section{Simultaneous linear regression analyses}

We conducted separate simultaneous linear regression analyses for each of the five OCI$\mathrm{R}$ subscales (checking, ordering, neutralizing, washing, and obsessing) and thus used a Bonferroni corrected critical $\alpha=.01$ (.05/ 5). Simultaneous linear regression models were chosen over hierarchical models because we did not have a-priori hypotheses regarding the change statistics of a model including AAQ-II and DTS scores relative to a model with only DASS-21 scores as statistical predictors. Further, model statistics presented in a simultaneous linear regression model are identical to those provided in the final step of a comprehensive hierarchical regression model, justifying the use of a simultaneous regression approach. Indices of multicollinearity were acceptable for all models (all tolerance values $\geq .55$ and all VIF $\leq 1.83$ ), suggesting a lack of redundancy in model predictors. Simultaneous linear regression statistics for each of the five models are presented in Table 3.

Checking symptoms. The overall regression model was significant and accounted for approximately $15.5 \%$ of variance in OCI-R checking scores, $F(3,465)=29.01, p<$ .001.DASS-21 scores significantly predicted checking symptoms above and beyond other predictors, accounting for $4.7 \%$ of OCI-R checking scores. Neither AAQ-II nor DTS significantly predicted checking, although there was a trend toward significance for AAQ-II scores.

Ordering symptoms. The overall regression model was significant and accounted for approximately $5.9 \%$ of variance in OCI-R ordering scores, $F(3,471)=9.79, p<.001$. Neither DASS-21, AAQ-II, nor DTS significantly predicted ordering, although there was a trend toward significance for DASS-21 scores.

Neutralizing symptoms. The overall regression model was significant and accounted for approximately $7.8 \%$ of variance in OCI-R neutralizing scores, $F(3,473)=13.26, p<$ 
Table 3. Simultaneous linear regressions predicting OC symptoms

\begin{tabular}{|c|c|c|c|c|c|c|}
\hline & $B$ & $S E_{B}$ & $\beta$ & $t$ & $p$ & $s p r^{2}$ \\
\hline \multicolumn{7}{|c|}{ Checking symptoms } \\
\hline DASS-21 & .06 & .02 & .26 & 4.87 & $<.001$ & .04 \\
\hline AAQ-II & .04 & .02 & .14 & 2.46 & .014 & .01 \\
\hline DTS & -.01 & .02 & -.05 & -.90 & .370 & $<.01$ \\
\hline \multicolumn{7}{|c|}{ Ordering symptoms } \\
\hline DASS-21 & .04 & .02 & .13 & 2.26 & .024 & .01 \\
\hline AAQ-II & .03 & .02 & .09 & 1.44 & .151 & $<.01$ \\
\hline DTS & -.02 & .01 & -.07 & -1.26 & .207 & $<.01$ \\
\hline \multicolumn{7}{|c|}{ Neutralizing symptoms } \\
\hline DASS-21 & .05 & .01 & .24 & 4.17 & $<.001$ & .03 \\
\hline AAQ-II & .00 & .01 & -.01 & -.15 & .878 & $<.01$ \\
\hline DTS & -.01 & .01 & -.08 & -1.43 & .153 & $<.01$ \\
\hline \multicolumn{7}{|c|}{ Washing symptoms } \\
\hline DASS-21 & .02 & .01 & .07 & 1.23 & .220 & $<.01$ \\
\hline AAQ-II & .03 & .02 & .10 & 1.59 & .112 & .01 \\
\hline DTS & -.01 & .01 & -.05 & -.97 & .331 & $<.01$ \\
\hline \multicolumn{7}{|c|}{ Obsessing symptoms } \\
\hline DASS-21 & .06 & .01 & .23 & 4.81 & $<.001$ & .03 \\
\hline AAQ-II & .10 & .01 & .37 & 7.22 & $<.001$ & .07 \\
\hline DTS & -.01 & .01 & -.05 & -1.15 & .251 & $<.01$ \\
\hline
\end{tabular}

Notes: DASS-21 = Depression Anxiety Stress Scales-21; AAQ-II = Acceptance and Action Questionnaire-II; DTS = Distress Tolerance Scale; $s p r^{2}=$ squared semipartial correlation. Bolded text indicates statistical significance reached after applying Bonferroni corrections controlling for multiple comparisons

.001. DASS-21 scores significantly predicted neutralizing symptoms above and beyond other predictors, accounting for $3.8 \%$ of OCI-R neutralizing scores. Neither AAQ-II nor DTS significantly predicted neutralizing.

Washing symptoms. The overall regression model was significant and accounted for approximately $3.5 \%$ of variance in OCI-R washing scores, $F(3,476)=5.79, p=.001$. Neither DASS-21, AAQ-II, nor DTS significantly predicted washing.

Obsessing symptoms. The overall regression model was significant and accounted for approximately $33 \%$ of variance in OCI-R obsessing scores, $F(3,473)=77.80, p<$ .001. DASS-21 scores significantly predicted obsessing symptoms above and beyond other predictors, accounting for $3.6 \%$ of OCI-R obsessing scores. AAQ-II scores also significantly predicted obsessing symptoms above and beyond other predictors, accounting for $8.5 \%$ of OCI-R obsessing scores. DTS did not significantly predict obsessing.

\section{Discussion}

Cognitive-behavioral models of OC symptoms have strong empirical support (e.g. Salkovskis, 1999). Nevertheless, obsessional beliefs only partially explain OC symptoms. Accordingly, it is worthwhile to examine other phenomena of interest that seem conceptually relevant to OCD 
and that might enhance or inform existing theoretical models. The current study sought to examine the unique contributions of EA and DT in the prediction of OC symptom dimensions. The present work builds on previous studies that have examined these constructs separately and is the first to simultaneously account for both IIE indices in the prediction of the various OC symptom dimensions.

Overall, our results suggest significant - if modest - associations among OC symptoms, EA, and DT in the expected directions, affirming the conceptual relatedness of these constructs. Yet, it appears to be one's willingness (i.e. psychological flexibility, EA) rather than ability (i.e. distress tolerance, DT) to sustain unpleasant internal experiences that best predicts obsessional symptoms above and beyond general distress. This finding highlights the distinction between simply enduring aversive emotions (i.e. DT; "I can tolerate being distressed or upset as well as most people") versus being willing and open to experiencing them (i.e. psychological flexibility; "It is okay if I remember something unpleasant"). Acceptance involves welcoming unwanted obsessional thoughts, images, and anxiety without attempting to change them versus simply tolerating them until they dissipate. This distinction is noteworthy because researchers have recently cautioned that over-reliance on fear reduction strategies (i.e. trying to eliminate unwanted internal experiences) may have unintended negative consequences that inadvertently maintain OC symptoms; specifically, individuals strive to control their anxiety rather than learning that anxiety itself is not threatening (Abramowitz and Arch, 2014). Accordingly, based on the current findings and their conceptual implications, researchers may wish to prioritize indices of EA (more so than DT) when conceptualizing, assessing, and treating OC symptoms.

Second, integrating this result with previous work (Cougle et al., 2011, 2012), obsessions may be the domain most robustly and consistently related to IIE. Indeed, obsessions often involve thoughts with unpleasant, ego-dystonic content (e.g. violence, morality) that (a) intrude frequently and abruptly into consciousness without identifiable evoking stimuli and (b) are associated with subtle mental rituals and neutralizing behaviors (Lee and Kwon, 2003). Given the relative ease and frequency with which mental rituals can be enacted, EA may especially contribute to the maintenance of unacceptable obsessions (as opposed, for example, to those pertaining to contamination or the need for exactness). Specifically, although our findings are merely correlational, it may be that individuals with high EA engage in repeated covert attempts to avoid or escape from frequent and unwanted internal experiences (i.e. unacceptable obsessional thoughts). These responses, in turn, might serve to exacerbate the obsessions, perhaps because the individual becomes hypervigilant for "bad thoughts" and preoccupied with eliminating them completely. Future research is necessary to directly test this possibility, however, especially in light of the fact that the OCI-R neutralizing scale used in the present study assesses more numerical neutralizing (e.g. "I feel I have to repeat certain numbers") than other mental thought control strategies.

It is worth mentioning that our IIE predictors accounted for a modest proportion of variance in OC symptom dimensions. Previous researchers have conjectured that the limited explanatory power of measures such as the AAQ is due to the fact that EA and DT are general measures of IIE (vs. other more disorder-specific constructs such as obsessive beliefs; Abramowitz et al., 2009). Individuals may find it difficult to report avoidance of thoughts and feelings considered generally (e.g. "Emotions cause problems in my life"), and may find it easier to report that they avoid internal events that are specifically associated with their condition of concern (e.g. "Unwanted intrusive thoughts cause problems in my life"). 
Accordingly, there have been an increasing number of versions of the AAQ that are being tailored to specific anxiety disorders (e.g. social anxiety; MacKenzie and Kocovski, 2010). We are currently developing a version of the AAQ tailored to OC symptoms that could advance the study of IIE, as other context-specific versions of the AAQ have demonstrated incremental validity over the general version of the AAQ (MacKenzie and Kocovski, 2010).

Along similar lines, future research on IIE would benefit from methodological consistency since, as previously mentioned, extant research proposes various conceptual and methodological differences that may account for mixed findings. First, the revised AAQII (Bond et al., 2011) is a psychometrically improved version of the original AAQ that demonstrates better psychometric consistency and should be prioritized over more traditional but outdated versions of the measure (until a psychometrically sound measure of OC-related EA becomes available). Second, given the heterogeneity of OC symptoms and findings suggesting that IIE is involved in some (i.e. obsessions), but not all, presentations of OC symptoms, researchers should utilize dimensional (versus global) OC symptom measures to capture the varying relationships between IIE and these symptom presentations. Finally, it is important that further work on predictive models test the relative contributions of EA and DT in predicting OC symptoms after accounting for obsessive beliefs, which have established predictive power in accounting for such symptoms. Moreover, it is also important to control for general negative affect considering that negative affect has been associated with DT, EA, and OCD symptoms. Such inclusive predictive models will provide a more accurate estimate of the explanatory power of IIE constructs.

To the extent that our non-clinical sample can be used to draw inferences about treatmentseeking individuals, the current study suggests that targeting EA, in particular, may have implications for the treatment of OCD. Acceptance-based approaches (e.g. ACT; Hayes, Luoma, Bond, Masuda and Lillis, 2006; Hayes, Strosahl and Wilson, 1999) use experiential metaphors to target EA in anxiety treatment, with preliminarily encouraging results (Twohig, Hayes and Masuda, 2006), and with additional research underway examining how such techniques might be integrated into established cognitive-behavioral interventions such as exposure and response prevention (Twohig et al., in press).

\section{Limitations}

A number of limitations of the present study should be noted. First, our data were collected using a non-clinical sample, which might not generalize to clinical samples. A recent review of the analogue literature, however, underscores the qualitative (but not quantitative) similarities between analogue and clinical OC symptoms (e.g. Abramowitz et al., 2014). Future research on this topic with clinical samples is therefore encouraged. Second, the reliance on self-report methodology might inflate associations among study measures, and is therefore a limitation. Third, the correlational nature of the study design prevents drawing causal conclusions. Although our findings are consistent with the theory that high levels of EA contribute to the development and maintenance of obsessional symptoms, an alternate explanation is that individuals with OCD develop maladaptive IIE difficulties as a result of their struggle with unacceptable thoughts. Fourth, our sample was predominantly young and white; therefore, we cannot speak to the generalizability of our findings to a population with greater demographic diversity. Finally, the omission of measures assessing constructs such as responsibility, thought-action fusion, and the need to control thoughts is a limitation in 
that it prevents conclusions regarding the relative explanatory power of IIE versus established cognitive variables (i.e. "obsessive beliefs"). As this is a key limitation of our study, future research should examine broader explanatory models incorporating both established and novel constructs.

\section{Acknowledgements}

Ethical standards: The authors assert that all procedures contributing to this work comply with the ethical standards of the relevant national and institutional committees on human experimentation and with the Helsinki Declaration of 1975, as revised in 2008.

Conflict of interest: The authors have no conflicts of interest with respect to this publication.

\section{References}

Abramowitz, J. S. and Arch, J. J. (2014). Strategies for improving long-term outcomes in cognitive behavioral therapy for obsessive-compulsive disorder: insights from learning theory. Cognitive and Behavioral Practice, 21, 20-31. http://doi.org/10.1016/j.cbpra.2013.06.004

Abramowitz, J. S., Fabricant, L. E., Taylor, S., Deacon, B. J., McKay, D. and Storch, E. A. (2014). The relevance of analogue studies for understanding obsessions and compulsions. Clinical Psychology Review, 34, 206-217.doi: 10.1016/j.cpr.2014.01.004

Abramowitz, J. S., Lackey, G. R. and Wheaton, M. G. (2009). Obsessive-compulsive symptoms: the contribution of obsessional beliefs and experiential avoidance. Journal of Anxiety Disorders, 23, 160166. doi:10.1016/j.janxdis.2008.06.003

American Psychiatric Association (2013). Diagnostic and Statistical Manual of Mental Disorders (5th ed.). Washington, DC: Author.

Antony, M. M., Bieling, P. J., Cox, B. J., Enns, M. W. and Swinson, R. P. (1998). Psychometric properties of the 42-item and 21-item versions of the Depression Anxiety Stress Scales in clinical groups and a community sample. Psychological Assessment, 10, 176-181.

Bernstein, A., Zvolensky, M. J., Vujanovic, A. A. and Moos, R. (2009). Integrating anxiety sensitivity, distress tolerance, and discomfort intolerance: a hierarchical model of affect sensitivity and tolerance. Behavior Therapy, 40, 291-301. doi:10.1016/j.beth.2008.08.001

Bond, F. W., Hayes, S. C., Baer, R. A., Carpenter, K. M., Guenole, N., Orcutt, H. K., et al. (2011). Preliminary psychometric properties of the Acceptance and Action Questionnaire-II: a revised measure of psychological inflexibility and experiential avoidance. Behavior Therapy, 42, 676-688. doi:10.1016/j.beth.2011.03.007

Briggs, E. S. and Price, I. R. (2009). The relationship between adverse childhood experience and obsessive-compulsive symptoms and beliefs: the role of anxiety, depression, and experiential avoidance. Journal of Anxiety Disorders, 23, 1037-1046. doi:10.1016/j.janxdis.2009.07.004

Chawla, N. and Ostafin, B. (2007). Experiential avoidance as a functional dimensional approach to psychopathology: an empirical review. Journal of Clinical Psychology, 63, 871-890. doi:10.1002/jclp.20400

Cougle, J. R., Timpano, K. R., Fitch, K. E. and Hawkins, K. A. (2011). Distress tolerance and obsessions: an integrative analysis. Depression and Anxiety, 28, 906-914. doi:10.1002/da.20846

Cougle, J. R., Timpano, K. R. and Goetz, A. R. (2012). Exploring the unique and interactive roles of distress tolerance and negative urgency in obsessions. Personality and Individual Differences, 52, 515-520. doi:10.1016/j.paid.2011.11.017 
Foa, E. B., Huppert, J. D., Leiberg, S., Langer, R., Kichic, R., Hajcak, G., et al. (2002). The Obsessive-Compulsive Inventory: development and validation of a short version. Psychological Assessment, 14, 485-496. doi:10.1037/1040-3590.14.4.485

Forsyth, J. P., Eifert, G. H. and Barrios, V. (2006). Fear conditioning in an emotion regulation context: a fresh perspective on the origins of anxiety disorders. In M. G. Craske, D. Hermans and D. Vansteenwegen (Eds.), Fear and Learning: from basic processes to clinical implications (pp. 133153). Washington, DC: American Psychological Association.

Hayes, S. C., Luoma, J. B., Bond, F. W., Masuda, A. and Lillis, J. (2006). Acceptance and commitment therapy: model, processes and outcomes. Behaviour Research and Therapy, 44, $1-25$.

Hayes, S. C., Strosahl, K. D. and Wilson, K. G. (1999). Acceptance and Commitment Therapy: an experiential approach to behavior change. New York: Guilford Press.

Hayes, S. C., Wilson, K. G., Gifford, E. V., Follette, V. M. and Strosahl, K. (1996). Experiential avoidance and behavioral disorders: a functional dimensional approach to diagnosis and treatment. Journal of Consulting and Clinical Psychology, 64, 1152-1168. doi:10.1037/0022-006X.64.6.1152

Henry, J. D. and Crawford, J. R. (2005). The short-form version of the Depression Anxiety Stress Scales (DASS-21): construct validity and normative data in a large non-clinical sample. British Journal of Clinical Psychology, 44, 227-239.

Hezel, D. M., Riemann, B. C. and McNally, R. J. (2012). Emotional distress and pain tolerance in obsessive-compulsive disorder. Journal of Behavior Therapy and Experimental Psychiatry, 43, 981987. doi:10.1016/j.jbtep.2012.03.005

Keough, M. E., Riccardi, C. J., Timpano, K. R., Mitchell, M. A. and Schmidt, N. B. (2010). Anxiety symptomatology: the association with distress tolerance and anxiety sensitivity. Behavior Therapy, 41, 567-574. doi:10.1016/j.beth.2010.04.002

Kline, R. B. (2005). Principles and Practices of Structural Equation Modeling (2nd edition). New York: Guilford Press.

Lee, H. J. and Kwon, S. M. (2003). Two different types of obsession: autogenous obsessions and reactive obsessions. Behaviour Research and Therapy, 41, 11-29. doi:10.1016/S0005-7967(01)00101-2

Leyro, T. M., Zvolensky, M. J. and Bernstein, A. (2010). Distress tolerance and psychopathological symptoms and disorders: a review of the empirical literature among adults. Psychological Bulletin, 136, 576-600. doi:10.1037/a0019712

Lovibond, S. H. and Lovibond, P. F. (1995). Manual for the Depression Anxiety Stress Scales (2nd ed.) Sydney: Psychology Foundation.

Macatee, R. J., Capron, D. W., Schmidt, N. B. and Cougle, J. R. (2013). An examination of low distress tolerance and life stressors as factors underlying obsessions. Journal of Psychiatric Research, 47, 1462-1468. doi:10.1016/j.jpsychires.2013.06.019

MacKenzie, M. B. and Kocovski, N. L. (2010). Self-reported acceptance of social anxiety symptoms: development and validation of the Social Anxiety - Acceptance and Action Questionnaire. International Journal of Behavioral Consultation and Therapy, 6, 214232.

Manos, R. C., Cahill, S. P., Wetterneck, C. T., Conelea, C. A., Ross, A. R. and Riemann, B. C. (2010). The impact of experiential avoidance and obsessive beliefs on obsessivecompulsive symptoms in a severe clinical sample. Journal of Anxiety Disorders, 24, 700-708. doi:10.1016/j.janxdis.2010.05.001

Obsessive Compulsive Cognitions Working Group (OCCWG) (1997). Cognitive assessment of obsessive-compulsive disorder. Behaviour Research and Therapy, 35, 667-681. doi:10.1016/S0005-7967(97)00017-X

Obsessive Compulsive Cognitions Working Group (OCCWG) (2001). Development and initial validation of the Obsessive Beliefs Questionnaire and the interpretation of intrusions inventory. Behaviour Research and Therapy, 39, 987-1006. doi:10.1016/S0005-7967(00)00085-1 
Obsessive Compulsive Cognitions Working Group (OCCWG) (2003). Psychometric validation of the Obsessive Beliefs Questionnaire and the Interpretation of Intrusions Inventory: Part I. Behaviour Research and Therapy, 41, 863. doi:10.1016/S0005-7967(02)00099-2

Obsessive Compulsive Cognitions Group Working (OCCWG) (2005). Psychometric validation of the Obsessive Belief Questionnaire and Interpretation of Intrusions Inventory. Part 2. Factor analyses and testing of a brief version. Behaviour Research and Therapy, 43, 1527-1542. doi:10.1016/j.brat.2004.07.010

Page, A. C. Hooke, G. R. and Morrison, D. L. (2007). Psychometric properties of the Depression Anxiety Stress Scales (DASS) in depressed clinical samples. British Journal of Clinical Psychology, 46, 283-297.

Rachman, S. J. and de Silva, P. (1978). Abnormal and normal obsessions. Behaviour Research and Therapy, 16, 233-248. doi:10.1016/0005-7967(78)90022-0

Ricciardi, J. N. and McNally, R. J. (1995). Depressed mood is related to obsessions, but not to compulsions, in obsessive-compulsive disorder. Journal of Anxiety Disorders, 9, 249-256. doi:10.1016/0887-6185(95)00006-A

Robinson, L. J. and Freeston, M. H. (2014). Emotion and internal experience in Obsessive Compulsive Disorder: reviewing the role of alexithymia, anxiety sensitivity and distress tolerance. Clinical Psychology Review, 34, 256-271. doi:10.1016/j.cpr.2014.03.003

Salkovskis, P. M. (1999). Understanding and treating obsessive-compulsive disorder. Behaviour Research and Therapy, 37 (Suppl 1), S29-S52. doi:10.1016/S0005-7967(99)00049-2

Salkovskis, P. M. and Harrison, J. (1984). Abnormal and normal obsessions: a replication. Behaviour Research and Therapy, 22, 549-552. doi:10.1016/0005-7967(84)90057-3

Simons, J. S. and Gaher, R. M. (2005). The Distress Tolerance Scale: development and validation of a self-report measure. Motivation and Emotion, 29, 83-102. doi:10.1007/s11031-005-7955-3

Teasdale, J. D. (1974). Learning models of obsessive-compulsive disorder. In H. R. Beech (Ed.), Obsessional States. London: Methuen.

Twohig, M. (2009). The application of Acceptance and Commitment Therapy to obsessive-compulsive disorder. Cognitive and Behavioral Practice, 16, 18-28. doi: 10.1016/j.cbpra. 2008.02.008.

Twohig, M. P., Abramowitz, J. S., Bluett, E. J., Fabricant, L. E., Jacoby, R. J., Morrison, K. L., et al. (in press). Exposure therapy for OCD from an acceptance and commitment therapy framework. Journal of Obsessive-Compulsive and Related Disorders. doi:10.1016/j.jocrd.2014.12.007

Twohig, M. P., Hayes, S. C. and Masuda, A. (2006). Increasing willingness to experience obsessions: acceptance and commitment therapy as a treatment for obsessive compulsive disorder. Behavior Therapy, 37, 3-13.doi:10.1016/j.beth.2005.02.001

Wetterneck, C. T., Steinberg, D. S. and Hart, J. (2014). Experiential avoidance in symptom dimensions of OCD. Bulletin of the Menninger Clinic, 78, 253-269. doi:10.1521/bumc.2014.78.3.253

Wolgast, M. (2014). What does the Acceptance and Action Questionnaire (AAQ-II) really measure? Behavior Therapy, 45, 831-839. doi:10.1016/j.beth.2014.07.002

Wu, K. D. and Carter, S. A. (2008). Further investigation of the Obsessive Beliefs Questionnaire: factor structure and specificity of relations with OCD symptoms. Journal of Anxiety Disorders, 22, 824-836. doi:10.1016/j.janxdis.2007.08.008 